\title{
A NEWMAN PROPERTY FOR BLD-MAPPINGS
}

\author{
RAMI LUISTO
}

\begin{abstract}
We define a Newman property for BLD-mappings and prove that for a BLD-mapping between generalized manifolds equipped with complete path-metrics, this property is equivalent to the branch set being porous when the codomain is LLC.
\end{abstract}

\section{INTRODUCTION}

The class of BLD-mappings was introduced by Martio and Väisälä in MV88 as a subclass of quasiregular mappings that preserve solutions of certain elliptic partial differential equations. In that paper Martio and Väisälä showed, among other results, that the class of BLD-mappings has several equivalent definitions. In this paper we use the following geometric definition applicable in any path-metric space. For the definitions of a branched cover, the length of a path and path-metric spaces, see Section 2. Given $L \geq 1$, a branched cover $f: M \rightarrow N$ between metric spaces is a mapping of Bounded Length Distortion, or $(L-) B L D$ for short, if for all paths $\gamma:[0,1] \rightarrow X$, we have

$$
L^{-1} \ell(\gamma) \leq \ell(f \circ \gamma) \leq L \ell(\gamma)
$$

We denote by $B_{f}$ the branch set of a branched cover $f$, i.e., the set of points in the domain where the mapping fails to be a local homeomorphism. A basic example of a BLD-mapping is the planar winding map $w: \mathbb{C} \rightarrow \mathbb{C}, z \mapsto \frac{z^{2}}{|z|}$ along with its higher-dimensional analogues $w \times \operatorname{id}_{\mathbb{R}^{n}}: \mathbb{R}^{n+2} \rightarrow \mathbb{R}^{n+2}$. These examples have branch sets of both zero measure and small topological dimension; such a property holds in general for BLD-mappings. Indeed, for an $L$-BLD mapping $f$ between $n$-dimensional Euclidean domains the topological dimension of the branch set is at most $n-2$ by the Cernavskii-Väisälä theorem (see, e.g., [Väi66]) and $\operatorname{dim}_{\mathcal{H}}\left(B_{f}\right)<$ $n-\varepsilon(n, L)$ (see, e.g., [BH04] and [MV88, Section 4.27]). The topological dimension of the branch set is not completely understood, even in dimension three; for example the Church-Hemmingsen conjecture in [CH60 asks when the Cernavskii-Väisälä theorem is exact. On the other hand, the estimate for the Hausdorff dimension is strict in the sense that for any $n \geq 3$ and $\varepsilon>0$ there exists a BLD-mapping $g: \mathbb{R}^{n} \rightarrow \mathbb{R}^{n}$ with $\operatorname{dim}_{\mathcal{H}}\left(B_{g}\right) \geq n-\varepsilon$; see, e.g., [HR02] and [MV88, Section 4.27].

With the Hausdorff dimension of the branch set bounded away from $n$, it follows that the branch set of a BLD-mapping $f: \Omega \rightarrow \mathbb{R}^{n}$ has $n$-measure zero. It is an interesting question, posed for example by Heinonen and Rickman in HR02, Remarks 6.7 (b)], that in what generality does this hold. The measure of the branch set is

Received by the editors November 7, 2017, and, in revised form, November 16, 2018.

2010 Mathematics Subject Classification. Primary 30L10, 30C65, 57M12.

The first author was partially supported by a grant of the Finnish Academy of Science and Letters, the Academy of Finland (grant 288501 "Geometry of subRiemannian groups"), and by the European Research Council (ERC Starting Grant 713998 GeoMeG "Geometry of Metric Groups"). 
also connected to certain limit properties for BLD-mappings; see [Lui17, Remark 4.5] for commentary on the topic. There are positive results concerning this question for BLD-mappings outside the setting of Riemannian manifolds; Heinonen and Rickman show in [HR02, Theorem 6.4] that for BLD-mappings between quasiconvex spaces of type A (see Section 2 for definitions) the branch set of a BLD-mapping has measure zero. It is not completely known which assumptions of HR02, Theorem 6.4] can be relaxed - especially if, in addition, the spaces are assumed to be generalized manifolds. We construct in Section 3.1 a BLD-mapping between Ahlfors 2-regular metric spaces with $\mathcal{H}^{2}\left(B_{f}\right)>0$ which exemplifies some of the restrictions for possible positive results in the future and the limitations of the methods in the current paper.

Our approach for the study of the measure theoretic size of the branch set is motivated by the fact that for a BLD-mapping $f: \Omega \rightarrow \mathbb{R}^{n}$ the branch set is locally porous, i.e., for any point $x_{0} \in B_{f}$ there exists a constant $\delta>0$ and a neighborhood $U$ of $x_{0}$ such that for any ball $B(x, r) \subset U$ there is a point $y \in B(x, r)$ for which $B_{f} \cap B(y, \delta r)=\emptyset$. In an Ahlfors $n$-regular metric measure space a porous set has measure zero, so in such a setting the question of Heinonen and Rickman can be approached via the porosity of the branch set. This approach has had previous success; in OR09] Onninen and Rajala study the branch set of quasiregular mappings between generalized manifolds of type A via a stratification to porous sets and reach estimates on the Hausdorff dimension of the branch set. Our approach to this question in the setting of BLD-mappings is motivated by the proof of MV88, Theorem 4.25]; the theorem in question implies that the branch set of a Euclidean BLD-mapping is porous. It turns out that the porosity is connected to the following concept of a Newman property for a BLD-mapping: $f$ satisfies an $\varepsilon_{0}$-Newman type property at $x_{0} \in X$ if there is a neighborhood $U$ of $x_{0}$ and a constant $\varepsilon_{0}>0$ such that in any ball $B(z, r) \subset U$ with $z \in B_{f}$, there exists a point $w \in B(z, r)$ such that

$$
\operatorname{diam}\left(B(z, r) \cap f^{-1}\{f(w)\}\right) \geq \varepsilon_{0} r .
$$

The term Newman property is motivated by an injectivity radius result of Newman from 1931, New31, which essentially states that if a finite group $G$ acts nontrivially on a connected topological manifold $M$, then at least one of the orbits has a diameter of at least $\varepsilon=\varepsilon(M)>0$. Newman's result was generalized by McAuley and Robinson in MR83 to state that for any manifold $M$ there is a constant $\varepsilon>0$ such that any surjective branched cover $f: M \rightarrow N$ onto a manifold $N$ is either a homeomorphism or has a fiber with a diameter of at least $\varepsilon$. Later on in MR84] they elaborated on the topic and coined the term Newman property; note, however, that our terminology differs slightly from theirs.

For BLD-mappings the Newman property is strongly connected to the porosity of the branch set and our main theorem is as follows. For the definition of the terms see Section 2 .

Theorem 1.1. Let $f: M \rightarrow N$ be an $L$-BLD-mapping between generalized $n$ manifolds both equipped with a complete path-metric. Suppose that the mapping $f$ satisfies a Newman property at $x_{0} \in M$. Then the branch set of $f$ is locally porous at $x_{0}$.

Furthermore if $N$ is $L L C$, then these conditions are equivalent.

We note that natural examples of generalized manifolds satisfying the local LLC condition arise from the classes of Riemannian and subRiemannian manifolds (see 
Section 21). The theory of BLD-mappings from a generalized manifold onto Euclidean spaces and manifolds have been studied, e.g., by Heinonen and Rickman in [HR02, and we record the following corollary in this spirit.

Corollary 1.2. Let $f: M \rightarrow N$ be an $L$-BLD-mapping where $M$ is a generalized $n$-manifold and $N$ a (sub)Riemannian $n$-manifold, both equipped with a complete path-metric. Then the following are equivalent for $x_{0} \in M$ :

(1) The mapping $f$ satisfies a Newman property at $x_{0}$.

(2) The branch set of $f$ is locally porous at $x_{0}$.

Finally, we wish to note that Newman-type properties have seen previous use in the study of geometric mappings in the form of the McAuley-Robinson theorem in [MR84; see, e.g., in Raj04 and [KOR03]. Furthermore, recent work of Guo and Williams partly relies on a generalization of the McAuley-Robinson theorem. This generalization, GWb, Corollary 4.8], is essentially a McAuley-Robinson theorem for branched covers between generalized manifolds with the extra assumptions that the domain satisfies a so-called $n$-LLC property and the codomain has bounded turning. Using this they show that for a BLD-mapping $f: M \rightarrow N$ between generalized $n$-manifolds equipped with Ahlfors regular measures the branch set has zero measure under the aforementioned additional assumptions of $M$ being $n$-LLC and $N$ having bounded turning. Beyond the use of a McAuley-Robinson type theorem their methods are quite dissimilar compared to the current manuscript; see GWa] and $[\mathrm{GWb}]$ for details.

\section{Preliminaries}

A mapping between topological spaces is said to be open if the image of every open set is open and discrete if the point inverses are discrete sets in the domain. A continuous, discrete and open mapping is called a branched cover. We follow the conventions of Ric93 and say that $U \subset X$ is a normal domain (for $f$ ) if $U$ is a precompact domain such that $\partial f(U)=f(\partial U)$. A normal domain $U$ is $a$ normal neighborhood of $x \in U$ if $\bar{U} \cap f^{-1}\{f(x)\}=\{x\}$. By $U(x, f, r)$ we denote the component of the open set $f^{-1} B_{Y}(f(x), r)$ containing $x$. The following lemma guarantees the existence of normal domains; for a proof see, e.g., Ric93, Lemma I.4.9, p. 19] or [Väi66, Lemma 5.1].

Lemma 2.1. Suppose $f: X \rightarrow Y$ is a branched cover between two locally compact and complete metric spaces. Then for every point $x \in X$ there exists a radius $r_{x}>0$ such that $U(x, f, r)$ is a normal neighborhood of $x$ for any $r \in\left(0, r_{x}\right)$.

Another topological result we need states that the branch set cannot have interior points in a very general setting. The following lemma can be found, e.g., from Väi66, Theorem 3.2].

Lemma 2.2. Suppose $f: X \rightarrow Y$ is a branched cover between two locally compact and complete metric spaces. Then the branch set $B_{f}$ has no interior points.

In a metric space $X$ the length $\ell(\gamma)$ of a path $\gamma:[0,1] \rightarrow X$ is defined as

$$
\ell(\gamma):=\left\{\sum_{j=1}^{k} d\left(\gamma\left(t_{j-1}\right), \gamma\left(t_{j}\right)\right) \mid 0=t_{0} \leq \cdots \leq t_{k}=1\right\} .
$$


Paths with finite and infinite length are called rectifiable and unrectifiable, respectively, and a metric space $(X, d)$ is a path-metric space if

$$
d(x, y)=\inf \{\ell(\gamma) \mid \gamma:[0,1] \rightarrow X, \gamma(0)=x, \gamma(1)=y\}
$$

for all $x, y \in X$. Similarly, a metric space $(X, d)$ is $(C$-) quasiconvex if for all $x, y \in X$ there exist a path $\gamma:[0,1] \rightarrow X$ with $\gamma(0)=x, \gamma(1)=y$ and $\ell(\gamma) \leq C d(x, y)$. A 1-quasiconvex space is called a geodesic space.

The following proposition is a part of Theorem 1.1 in Lui17.

Proposition 2.3. Let $f: M \rightarrow N$ be a continuous mapping between two complete locally compact path-metric spaces and $L \geq 1$. Then the following are equivalent:

(i) $f$ is an $L$-BLD-mapping and

(ii) $f$ is a discrete $L$-LQ-mapping.

Here a mapping $f: M \rightarrow N$ is L-Lipschitz Quotient, L-LQ for short, if for all $x \in X$ and $r>0$ we have

$$
B\left(f(x), L^{-1} r\right) \subset f B(x, r) \subset B(f(x), L r) .
$$

We will use the fact that $L$-BLD-mappings are $L$-LQ maps repeatedly in the arguments.

A natural setting for the study of branched covers is that of generalized manifolds; see [HR02, Section I.1] for details.

Definition 2.4. A locally compact Hausdorff space $M$ which is both connected and locally connected is called a generalized $n$-manifold if

(1) the cohomological dimension $\operatorname{dim}_{\mathbb{Z}} M$ is at most $n$ and

(2) the local cohomology groups of $M$ are equal to $\mathbb{Z}$ in degree $n$ and zero in degree $n-1$.

The importance of generalized manifolds arises from the fact that for a branched cover $f: M \rightarrow N$ between generalized $n$-manifolds topological index and degree theory are applicable. Especially, topological index and degree theory imply the following lemma; see, e.g., Ric93, Proposition I.4.10 (1)] for a proof. Here we denote by $N(y, f, U)$ the amount of preimages of $y$ under $f$ in $U$, and set $N(f, U)$ to be the maximum of $y \mapsto N(y, f, U)$ in $U$.

Lemma 2.5. Let $f: M \rightarrow N$ be a branched cover between two generalized $n$ manifolds. Then for any normal domain $U \subset M$, every point $y \in f U \backslash f B_{f}$ satisfies $N(y, f, U)=N(f, U)<\infty$.

For BLD-mappings between generalized manifolds the amount of preimages can be quantitatively bounded in some cases. This in turn gives rise to further geometrical control for the mapping. The following lemma is a variation of known family of results in this vein; compare, e.g., to [MV88, Theorems 4.12 and 4.14], [LP, Lemma 3.2], and [Lui16, Theorem 1.4]. We give a short proof for the convenience of the reader.

Lemma 2.6. Let $f: M \rightarrow N$ be an $L$-BLD-mapping between two generalized $n$ manifolds equipped with complete path-metrics, and let $U \subset M$ be a normal domain. Then for any $r_{0}>0$ and any continuum $K \subset f U$ with $B\left(K, r_{0}\right) \subset f U$ and $(\operatorname{diam} K)<r_{0}$, all the components of $U \cap f^{-1} K$ have a diameter of at most $2 L(N(f, U)+1)(\operatorname{diam} K)$. 
Proof. Fix a continuum $K \subset f U$ with $B\left(K, r_{0}\right) \subset f U$ and $\operatorname{diam} K<r_{0}$ for some $r_{0}>0$. Take a component $C$ of $U \cap f^{-1} K$ and note that if $\operatorname{diam} C>2 L(N(f, U)+$ $1)(\operatorname{diam} K)$, then there exists $k:=N(f, U)+1$ points $x_{1}, \ldots, x_{k} \in K$ such that $d\left(x_{i}, x_{j}\right)>2 L(\operatorname{diam} K)$ for any $i \neq j$.

Now we note that since $f$ is $L$-LQ, the images $f B\left(x_{i}, L(\operatorname{diam} K)\right)$ of the disjoint balls $B\left(x_{i}, L(\operatorname{diam} K)\right)$ all contain a ball of radius diam $K$. Since $f\left(x_{i}\right) \in K$ for each $i$, this implies that there exists a point $y_{0} \in \bigcap_{i=1}^{k} f B\left(x_{i}, L(\operatorname{diam} K)\right)$, and in particular $N\left(f, U, y_{0}\right) \geq k=N(f, U)+1$. This is a contradiction, and so the original claim holds.

Another crucial notion for us is local linear contractibility.

Definition 2.7. A metric space $X$ is locally linearly contractible (LLC) if for any compact set $K \subset X$ there exist constants $C \geq 1$ and $r_{0}>0$ such that for all $x \in K$ and $r \in\left(0, r_{0}\right)$ the ball $B(x, r) \subset X$ is contractible in $B(x, C r)$.

Natural examples of LLC spaces arise from Euclidean spaces, Riemannian manifolds (via the local bilipschitz equivalence of Riemannian manifolds and Euclidean spaces given by the exponential map), and subRiemannian manifolds (via the ballbox theorem; see, e.g., Mon02, Theorem 2.10, p. 29]).

In the literature the LLC condition is often used as a part of the definition of spaces of type $A$. We do not use spaces of type A either in the statement or in the proof of our main theorem, but describing the properties of the example constructed in Section 3.1 is more fluent with this standard terminology. We refer the reader to [HR02, Section I.1] for more details.

Definition 2.8. For a metric space $X$ and an integer $n \geq 2$ consider the following properties that $X$ may or may not have:

(A1) $X$ is $n$-rectifiable and has locally finite Hausdorff $n$-measure.

(A2) $X$ is $n$-Ahlfors regular.

(A3) $X$ is locally bilipschitz embeddable in a Euclidean space.

(A4) $X$ is LLC, i.e., locally linearly contractible.

A space $X$ is said to be of type $A$ if it satisfies all of these properties and of type (A123) if it satisfies properties (A1), (A2) and (A3).

The proofs of the main theorems rely on the concept of a blow up of a given metric space. The blow up of a metric space at a point can be seen as a generalization of a tangent space; indeed, in the setting of smooth manifolds, a blow up at a point $x_{0}$ is isometric to the tangent plane of the space at $x_{0}$. For the general theory of blow ups we refer to Kap09; for their interaction with BLD- and LQ-mappings see, e.g., [LP14, Section 3] and [Lui17, Section 4].

\section{MAIN RESUltS}

We begin by proving the first implication of the main theorem in the form of the following Proposition 3.1 .

Proposition 3.1. Let $f: M \rightarrow N$ be an $L$-BLD-mapping between two generalized manifolds both equipped with a complete path-metric. Suppose that $f$ satisfies an $\varepsilon_{0}$ Newman property at $x_{0}$. Then $B_{f}$ is locally porous at $x_{0}$. 
Proof. Suppose $B_{f}$ is not locally porous at $x_{0}$. Then by the definition of porosity we can fix a sequence $r_{n} \searrow 0$ such that

$$
B\left(x, \frac{1}{n} r_{n}\right) \cap B_{f} \neq \emptyset
$$

for all $x \in B\left(x_{0}, \frac{1}{2} r_{n}\right)$ and $n \in \mathbb{N}$. For each $j \in \mathbb{N}$, denote by $X_{j}$ and $Y_{j}$ the spaces $M$ and $N$ equipped with the metrics scaled by the factor $r_{j}^{-1}$, respectively. The $L$-BLD-mapping $f: M \rightarrow N$ induces canonical $L$-BLD-mappings $f_{j}: X_{j} \rightarrow Y_{j}$ for each $j \in \mathbb{N}$. Note that by (3.1) for all $j \in \mathbb{N}$ and $x \in B_{X_{j}}\left(x_{0}, \frac{1}{2}\right)$, we have $B_{X_{j}}\left(x, j^{-1}\right) \cap B_{f} \neq \emptyset$.

We refer to [Lui17, Section 4] for the fact that there exists, after possibly passing to a subsequence, locally compact and complete path-metric spaces $\left(\hat{X}, \hat{x}_{0}\right)$ and $\left(\hat{Y}, \hat{y}_{0}\right)$ together with an $L$-Lipschitz mapping $\hat{f}: \hat{X} \rightarrow \hat{Y}$ such that in the sense of convergence of mapping packages,

$$
\lim _{j \rightarrow \infty}\left(\left(X_{j}, x_{0}\right),\left(Y_{j}, f\left(x_{0}\right)\right), f_{j}\right)=\left(\left(\hat{X}, \hat{x}_{0}\right),\left(\hat{Y}, \hat{y}_{0}\right), \hat{f}\right)
$$

and $\hat{f}\left(\hat{x}_{0}\right)=\hat{y}_{0}$. Note that for any normal neighborhood $U$ of $x_{0}$ any point in $f U$ has at most finitely many preimages in $U$ since $f$ is discrete and $U$ is precompact. Thus by Lemma 2.5 there exists $N_{0} \in \mathbb{N}$ such that $U \cap f^{-1}\{f(x)\} \leq N_{0}$ for all $x \in U$. This implies that for any $r>0$ and all $j \in \mathbb{N}$ large enough,

$$
\#\left(B_{X_{j}}\left(x_{0}, r\right) \cap f^{-1}\{f(x)\}\right) \leq N_{0}
$$

for all $x \in X_{j}$. Thus the mappings $f_{j}: X_{j} \rightarrow Y_{j}$ have a uniform bound on their multiplicity, and by [Lui17, Theorem 4.1] the limiting map $\hat{f}: \hat{X} \rightarrow \hat{Y}$ is $L$-BLD. (In the proof of [Lui17, Theorem 4.1] the assumption about having spaces of type A with uniform constants is only used to guarantee a uniform multiplicity bound $(\mathrm{N})$, which we have here from (3.2).)

We next show that $B_{\hat{f}} \supset B_{\hat{X}}\left(\hat{x}_{0}, \frac{1}{4}\right)$. To this end, let $\hat{y} \in B_{\hat{X}}\left(\hat{x}_{0}, \frac{1}{4}\right)$. Let $\left(y_{j}\right)$ be a sequence in $\prod_{j} X_{j}$ corresponding to $\hat{y}$, and fix $\varepsilon \in(0,1 / 4)$. For each $j$ we study the ball $B_{j}:=B_{X_{j}}\left(y_{j}, \varepsilon\right)$. By the definition of the spaces $X_{j}$ and equation (3.1), there exists $b_{j} \in B_{f_{j}} \cap B_{j}$ for all large enough $j$. Now by the Newman property there exists a constant $\delta_{0}>0$ such that, for all $j$ large enough, there exists a point $z_{j} \in B_{j}$ for which

$$
\operatorname{diam}\left(B\left(b_{j}, \frac{1}{2}\right) \cap f_{j}^{-1}\left\{f\left(z_{j}\right)\right\}\right) \geq \delta_{0} .
$$

From these preimages of relatively large diameter we extract two sequences, $\left(w_{j}\right)$ and $\left(w_{j}^{\prime}\right)$ with $d_{X_{j}}\left(w_{j}, w_{j}^{\prime}\right) \geq \delta_{0}$ for all $j \in \mathbb{N}$. After passing to a subsequence if necessary, these sequences converge to points $\hat{w}, \hat{w}^{\prime} \in B(\hat{y}, \varepsilon) \subset \hat{X}$, respectively, such that $\hat{d}\left(\hat{w}, \hat{w}^{\prime}\right) \geq \delta_{0}>0$. Furthermore, the points $\hat{w}$ and $\hat{w}^{\prime}$ map to the same point under $\hat{f}$. Thus $\hat{f}$ is not injective in $B(\hat{y}, \varepsilon)$. Since the argument goes through for any $\varepsilon \in(0,1 / 4)$, we conclude that $\hat{y} \in B_{\hat{f}}$.

Thus the limit map $\hat{f}$, which is an $L$-BLD-mapping between locally compact and complete metric spaces, contains a ball in its branch set. This is a contradiction with Lemma 2.2 and the original claim holds.

We next show that the converse of Proposition 3.1 holds under the extra assumption that the space $N$ is LLC. 
The proof of Proposition 3.3 relies on the following Zorich-type result for BLDmappings which we have not seen explicitly stated in the literature, even though it is known to the experts in the field; for a Euclidean version see, e.g., MV88, Lemma $4.3]$.

Lemma 3.2. Let $f: M \rightarrow N$ be an $L$-BLD-mapping between generalized $n$-manifolds both equipped with a complete path-metric. Furthermore, suppose that $N$ is LLC. Then for each precompact domain $U \subset M$ there exist constants $r_{0}>0$ and $\phi>0$ such that if $B(x, r) \subset U$ and $B(x, r) \cap B_{f}=\emptyset$ for some $x \in X, r \in\left(0, r_{0}\right)$, then $\left.f\right|_{B(x, \phi r)}$ is injective.

Proof. Let $U \subset M$ be a precompact domain. Since $\bar{U}$ is compact it can be covered with finitely many normal neighborhoods $U\left(x_{j}, f, r_{j}\right)$. Thus by Lebesgue's number lemma there exists a radius $\delta>0$ such that any ball in $N$ of radius $\delta$ is contained in at least one of the balls $B\left(f\left(x_{j}\right), r_{j}\right)$. We may thus assume that $U$ itself is a normal neighborhood by requiring $r_{0}$ to be small enough. Let $D$ be the LLC-constant of the space $N$ with respect to the compact set $f \bar{U}$. We denote $k:=N(f, U)$ and set $\phi:=\left(16 L^{2}(k+1) D\right)^{-1}$; note that $\phi \leq 2^{-1}$. Finally let $r_{0}$ be so small that $2 \phi L r_{0}$ is below the LLC-radius of the space $N$ with respect to the compact set $f \bar{U}$.

Fix a ball $B\left(x_{0}, r\right) \subset U$ with $B\left(x_{0}, r\right) \cap B_{f}=\emptyset$ for some $x_{0} \in U$. Towards contradiction assume that there exist disjoint points $a, b \in B\left(x_{0}, \phi r\right)$ with $f(a)=$ $f(b)$. By the Hopf-Rinow theorem (see, e.g., Gro99, p. 9]) the locally compact and complete path-metric space $M$ is a geodesic space, and so we may fix a geodesic $\beta:[0,1] \rightarrow M$ with $\beta(0)=a, \beta(1)=b$. Note that $\operatorname{diam}|\beta| \leq 2 \phi r$, so $\beta:[0,1] \rightarrow U$. Since $f$ is $L$-Lipschitz,

$$
\operatorname{diam}(f|\beta|) \leq L \operatorname{diam}(|\beta|) \leq 2 L \phi r=\frac{r}{8 L(k+1) D}
$$

Since $f(a)=f(b), f \circ \beta:[0,1] \rightarrow B(f(a), 2 L \phi r)$ is a loop based on $f(a)$. Thus, be the local LLC-property of $N$, there is a homotopy $H:[0,1]^{2} \rightarrow B(f(a), 2 D L \phi r)$ taking the loop $f \circ \beta$ to a constant path $t \mapsto f(a)$.

Now by the path lifting theorem [Ric93, Theorem II.3.2] each of the paths $t \mapsto$ $H(t, s), s \in[0,1]$, has a unique total lift $\alpha_{s}$ in the normal domain $U$ starting from $a$. On the other hand we note that for each $s \in[0,1]$,

$$
\operatorname{diam}\left(f\left|\alpha_{s}\right|\right) \leq \operatorname{diam}\left(H\left([0,1]^{2}\right)\right) \leq 4 D L \phi r=\frac{r}{4 L(k+1)},
$$

and so by Lemma 2.6 each of the lifts $\alpha_{s}$ has diameter of at most

$$
2 L(k+1) \frac{r}{4 L(k+1)}=r / 2 .
$$

In particular, this implies that each of the lifts $\alpha_{s}$ is contained in the ball $B\left(x_{0}, r\right)$, since $a \in B\left(x_{0}, \phi r\right) \subset B\left(x_{0}, r / 2\right)$. Thus the lifts do not intersect any branch points. From this we conclude that the homotopy $H$ lifts to a homotopy $\tilde{H}$ in $B\left(x_{0}, r\right)$ contracting the path $\beta$ to a constant path while keeping the endpoints $a$ and $b$ fixed. This is a contradiction so the original claim holds true: $f$ is injective in $B(x, \phi r)$.

Proposition 3.3. Let $f: M \rightarrow N$ be an $L$-BLD-mapping between generalized $n$ manifolds equipped with a complete path-metric, and let $N$ be locally LLC. Suppose $B_{f}$ is locally porous at $x_{0} \in B_{f}$. Then $f$ satisfies a Newman property at $x_{0}$. 
Proof. Since $B_{f}$ is locally porous at $x_{0} \in B_{f}$, there exists by the definition of porosity a neighborhood $U$ of $x_{0}$ and a constant $\delta>0$ such that for all $x \in U$ with $B(x, r) \subset U$, there exists a point $y \in B(x, r)$ with

$$
B(y, \delta r) \subset B(x, r) \backslash B_{f} .
$$

Let $B(z, s) \subset U$ with $z \in B_{f}$. By the definition of the branch, $f$ cannot be locally injective at $z$. Thus by Lemmas 2.1 and 2.5 we may assume both that $U$ is a normal domain and that all points in $f U \backslash f B_{f}$ have an equal amount of preimages in $U$. Note that by the Cernavskii-Väisälä theorem (see [Väi66]) $f B_{f}$ has no interior points, and so its complement in $f U$ is not empty.

By the local porosity of $B_{f}$, there exists a point $w \in B\left(z,\left(2 L^{2}\right)^{-1} s\right)$ such that

$$
B\left(z, \delta(2 L)^{-1} s\right) \subset B\left(z,\left(2 L^{2}\right)^{-1} s\right) \backslash B_{f} .
$$

By Lemma 3.2 there exists a constant $\phi>0$ for which $\left.f\right|_{B\left(w, \phi \delta\left(2 L^{2}\right)^{-1} s\right)}$ is injective. The map $f$ is an open mapping, so the set $f B\left(w, \phi \delta\left(4 L^{2}\right)^{-1} s\right)$ is open and must contain a point $y \in f U \backslash f B_{f}$. Thus the point $y$ has at least two preimages in $U$, one of them in $B\left(w, \phi \delta\left(4 L^{2}\right)^{-1} s\right)$. We claim that there is another preimage of $y$ in the ball $B(z, s)$. Indeed, fix a geodesic $\beta$ connecting $y$ to $f(z)$. Since $f$ is $L$-BLD, the path $\beta$ has length of at most $(2 L)^{-1} s$. By the path-lifting theorem [Ric93, Theorem II.3.2], the path $\beta$ has at least two total lifts in $U$ starting from $z$ and these two lifts have disjoint endpoints in $f^{-1}\{y\}$ since $y \notin f B_{f}$. Furthermore, since $f$ is $L$-BLD, these two lifts have length of at most $2^{-1} s$. Thus we have

$$
\operatorname{diam}\left(B(z, s) \cap f^{-1}\{y\}\right) \geq \phi \delta\left(4 L^{2}\right)^{-1} s
$$

since $\left.f\right|_{B\left(w, \phi \delta\left(2 L^{2}\right)^{-1} s\right)}$ is injective, and so $f$ has the Newman property at $x_{0}$.

Proof of Theorem 1.1. The proof of the theorem now follows by combining Propositions 3.1 and 3.3

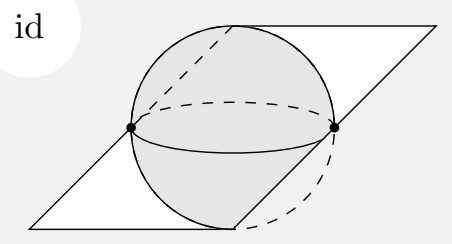

Figure 1. The auxiliary domain $W$ in the construction of the mapping in Example 3.4 .

3.1. A BLD-mapping with a branch set of positive measure. In this section we construct a compact and complete path-metric space $X$ equipped with an Ahlfors 2-regular measure together with a 2-BLD mapping $f: X \rightarrow X$ such that $\mathcal{H}^{2}\left(B_{f}\right)>0$. Furthermore, the constructed space $X$ is of type (A123) and contains an open dense set which is a 2-manifold, though it is not a generalized manifold as it has local cut-points. 
The construction of the space $X$ and the mapping $f: X \rightarrow X$ in this section is given as a limit of so-called pointed mapping packages. Even though for our purposes this terminology is a slightly excessive, it enables us to refer directly to previous work in Lui17. Here a pointed mapping package is a triple $\left(\left(X, x_{0}\right),\left(Y, y_{0}\right), f\right)$ where $X$ and $Y$ are locally compact and complete path-metric spaces having fixed base-points $x_{0} \in X, y_{0} \in Y$, and $f: X \rightarrow Y$ is a continuous mapping satisfying $f\left(x_{0}\right)=y_{0}$. For the definition of the convergence of a sequence of pointed mapping packages we refer to [DS97, Definition 8.18 and Lemma 8.19]; see also [KM, Definition 3.8] and Dav15, Definition 2.1]. In essence, the definition states that $\left(X_{j}, x_{j}\right) \rightarrow\left(X, x_{0}\right)$ and $\left(Y_{j}, y_{j}\right) \rightarrow\left(Y, y_{0}\right)$ in the Gromov-Hausdorff sense and $f_{j} \rightarrow f$ in a natural pointwise manner; indeed for constant sequences $\left(X_{j}, x_{j}\right)$ and $\left(Y_{j}, y_{j}\right)$ the convergence is just the pointwise convergence of mappings; see again, e.g., DS97.

Before constructing the sequence of pointed mapping packages, we define some auxiliary concepts. In the two-sphere $\mathbb{S}^{2}$ we define the winding map as the restriction of the winding map $w: \mathbb{R}^{n} \rightarrow \mathbb{R}^{n}, w(r, \theta, z)=(r, 2 \theta, z)$. Next we set

$$
W=\mathbb{S}^{2} \cup\left(\{0\} \times\left([-4,4]^{2} \backslash(-2,2) \times(-1,1)\right)\right) \subset \mathbb{R}^{3}
$$

(see Figure 1) and let $g: W \rightarrow W$ be a mapping which equals identity on the rectangular annulus, and the 2 -to-1 winding map on the sphere $\mathbb{S}^{2}$. In particular, we note that $g$ has the same "boundary data" as id: $[-4,4]^{2} \rightarrow[-4,4]^{2}$, even though these mappings are defined on different domains. Note also that the mapping $g$ has exactly two branch points at the poles of $\mathbb{S}^{2}$. Furthermore, a straightforward calculation shows that $g$ is 2-BLD.

Example 3.4. We define the sequence $\left(f_{j}, X_{j}, x_{j}, Y_{j}, y_{j}\right)$ recursively. Since for us $X_{j}=Y_{j}$ and $x_{j}=y_{j}=(0,0,0)$ for all $j \in \mathbb{N}$, we denote the mapping package as $\left(f_{j}, X_{j}\right)$ for brevity.

First we set $X_{0}=\{0\} \times \mathbb{R}^{2}$ and $f_{0}=\mathrm{id}: X_{0} \rightarrow X_{0}$. On $X_{0}$ we fix a sequence $\left(C_{j}\right)$ of nested collections of squares giving rise to a Sierpinski carpet of positive measure. We first set $C_{0}=\left\{\{0\} \times[0,1]^{2}\right\}$, and for each collection $C_{n}$ defined we take for every $K \in C_{n}$ four disjoint subsquares of $K$, each with area $\left(1-4^{-n-1}\right) \mathcal{H}^{2}(K)$ and positioned symmetrically in $K$ (see Figure 2). This collection of $4^{n+1}$ squares is then denoted $C_{n+1}$. With this construction the set

$$
C:=\bigcap_{n \in \mathbb{N}} \bigcup_{K \in C_{n}} K
$$

is a Cantor set of positive measure.

Suppose next that $\left(f_{n}, X_{n}\right)$ has been defined. We set $\left(f_{n+1}, X_{n+1}\right)$ equal $\left(f_{n}, X_{n}\right)$ outside the set $\bigcup C_{n+1}$. On each of the squares $S \in C_{n}$ we fix a subsquare $V$ around the center of $S$ such that $V \cap \bigcup C_{n+1}=\emptyset$. Finally, on each such square $V$ we replace the square $V$ and the identity mapping $\left.f_{n}\right|_{V}$ with a scaled version of the mapping $g: W \rightarrow W$; see again Figure 2,

The sequence $\left(f_{j}, X_{j}\right)$ as defined is a Cauchy sequence with respect to the Hausdorff-Gromov convergence of the sequence $\left(X_{j}\right)$ and the 2-BLD-mappings $\left(f_{j}\right)$ are 2-Lipschitz. Furthermore, the mappings $f_{j}$ are of uniformly bounded multiplicity, i.e., for any $f_{j}, j \in \mathbb{N}$, any given point has at most two preimages. Thus by the proof of Theorem 1.4 in Lui17 there exists a subsequence of this sequence converging to a 2-BLD-mapping $f: X \rightarrow X$, where $X$ is the Gromov-Hausdorff 


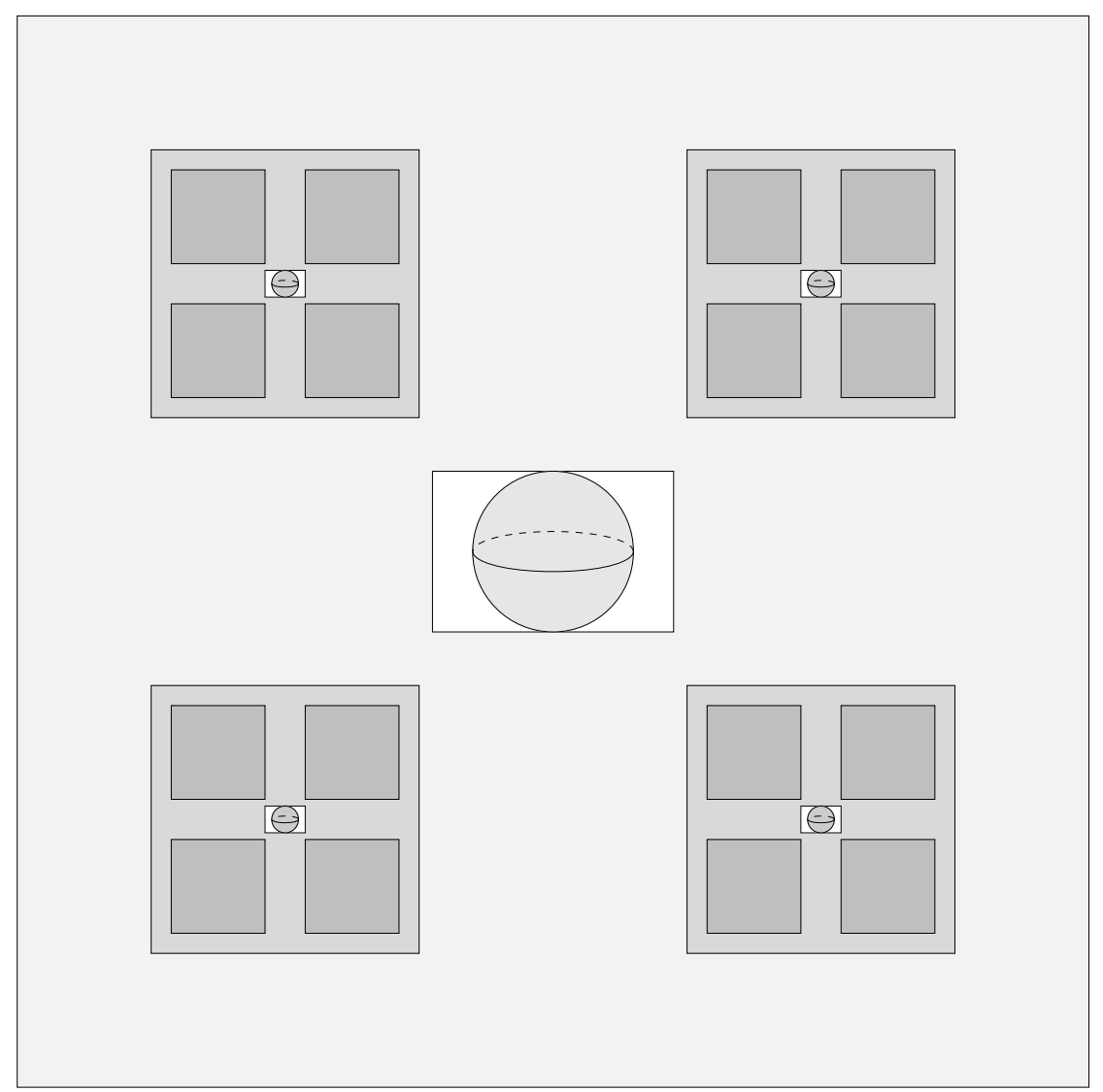

Figure 2. Cantor set modifications in Example 3.4 pictured is part of the domain of $f_{2}: X_{2} \rightarrow X_{2}$.

limit of $\left(X_{j}\right)$. Finally a moment's thought shows that the branch set of $f$ contains the Cantor set $C$ with $\mathcal{H}^{2}(C)>0$.

Remark 3.5. With minor modifications, the mapping in Example 3.4 can be modified to a 1-BLD-mapping $Y \rightarrow X$, where $Y$ is bilipschitz equivalent to $X$; indeed, this follows by pulling back the path length structure of the codomain under $f$; see, e.g., Aal16, Theorem 1.8].

\section{ACKNOWLEDGMENTS}

We thank Pekka Pankka for discussions on the topic. We also extend our thanks to the anonymous referee whose comments have improved the exposition of this manuscript. In particular, their suggestion for the proper generality of the main theorem is gratefully acknowledged. 


\section{REFERENCES}

[Aal16] Martina Aaltonen, Monodromy representations of completed coverings, Rev. Mat. Iberoam. 32 (2016), no. 2, 533-570, DOI 10.4171/RMI/894. MR.3512426

[BH04] Mario Bonk and Juha Heinonen, Smooth quasiregular mappings with branching, Publ. Math. Inst. Hautes Études Sci. 100 (2004), 153-170, DOI 10.1007/s10240-004-0024-8. MR:2102699

[CH60] Philip T. Church and Erik Hemmingsen, Light open maps on $n$-manifolds, Duke Math. J. 27 (1960), 527-536. MR 116315

[Dav15] Guy C. David, Tangents and rectifiability of Ahlfors regular Lipschitz differentiability spaces, Geom. Funct. Anal. 25 (2015), no. 2, 553-579, DOI 10.1007/s00039-015-0325-8. MR.3334235

[DS97] Guy David and Stephen Semmes, Fractured fractals and broken dreams: Self-similar geometry through metric and measure, Oxford Lecture Series in Mathematics and its Applications, vol. 7, The Clarendon Press, Oxford University Press, New York, 1997. MR.1616732

[Gro99] Misha Gromov, Metric structures for Riemannian and non-Riemannian spaces, Progress in Mathematics, vol. 152, Birkhäuser Boston, Inc., Boston, MA, 1999. Based on the 1981 French original [MR0682063 (85e:53051)]; With appendices by M. Katz, P. Pansu and S. Semmes; Translated from the French by Sean Michael Bates. MR:1699320

[GWa] Changyu Guo and Marshall Williams, Geometric function theory: the art of pullback factorization, preprint.

[GWb] Changyu Guo and Marshall Williams, Porosity of the branch set of discrete open mappings with controlled linear dilatation, preprint.

[HR02] Juha Heinonen and Seppo Rickman, Geometric branched covers between generalized manifolds, Duke Math. J. 113 (2002), no. 3, 465-529, DOI 10.1215/S0012-7094-0211333-7. MR 1909607

[Kap09] Michael Kapovich, Hyperbolic manifolds and discrete groups, Modern Birkhäuser Classics, Birkhäuser Boston, Inc., Boston, MA, 2009. Reprint of the 2001 edition. MR2553578

[KM] Bruce Kleiner and John M. Mackay, Differentiable structures on metric measure spaces: a primer, Ann. Sc. Norm. Super. Pisa Cl. Sci. (5) 16 (2016), no. 1, 41-64. MR.3524664

[KOR03] Pekka Koskela, Jani Onninen, and Kai Rajala, Mappings of finite distortion: injectivity radius of a local homeomorphism, Future trends in geometric function theory, Rep. Univ. Jyväskylä Dep. Math. Stat., vol. 92, Univ. Jyväskylä, Jyväskylä, 2003, pp. 169174. MR2061002

[LP] Rami Luisto and Pekka Pankka, Rigidity of extremal quasiregularly elliptic manifolds, Groups Geom. Dyn. 10 (2016), no. 2, 723-732, DOI 10.4171/GGD/362. MR3513114

[LP14] Enrico Le Donne and Pekka Pankka, Closed BLD-elliptic manifolds have virtually Abelian fundamental groups, New York J. Math. 20 (2014), 209-216. MR3177172

[Lui16] Rami Luisto, Note on local-to-global properties of BLD-mappings, Proc. Amer. Math. Soc. 144 (2016), no. 2, 599-607, DOI 10.1090/proc12711. MR3430837

[Lui17] Rami Luisto, A characterization of BLD-mappings between metric spaces, J. Geom. Anal. 27 (2017), no. 3, 2081-2097, DOI 10.1007/s12220-016-9752-5. MR3667422

[Mon02] Richard Montgomery, A tour of subriemannian geometries, their geodesics and applications, Mathematical Surveys and Monographs, vol. 91, American Mathematical Society, Providence, RI, 2002. MR1867362

[MR83] L. F. McAuley and Eric E. Robinson, On Newman's theorem for finite-to-one open mappings on manifolds, Proc. Amer. Math. Soc. 87 (1983), no. 3, 561-566, DOI 10.2307/2043652. MR684659

[MR84] Louis F. McAuley and Eric E. Robinson, Discrete open and closed mappings on generalized continua and Newman's property, Canad. J. Math. 36 (1984), no. 6, 1081-1112, DOI 10.4153/CJM-1984-062-9. MR771929

[MV88] O. Martio and J. Väisälä, Elliptic equations and maps of bounded length distortion, Math. Ann. 282 (1988), no. 3, 423-443, DOI 10.1007/BF01460043. MR967022

[New31] M. H. A. Newman, A theorem on periodic transformations of spaces, Q. J. Math., Oxf. Ser. 2 (1931), 1-8. 
[OR09] Jani Onninen and Kai Rajala, Quasiregular mappings to generalized manifolds, J. Anal. Math. 109 (2009), 33-79, DOI 10.1007/s11854-009-0028-x. MR2585391

[Raj04] Kai Rajala, A lower bound for the Bloch radius of K-quasiregular mappings, Proc. Amer. Math. Soc. 132 (2004), no. 9, 2593-2601, DOI 10.1090/S0002-9939-04-07405-2. MR 2054784

[Ric93] Seppo Rickman, Quasiregular mappings, Ergebnisse der Mathematik und ihrer Grenzgebiete (3) [Results in Mathematics and Related Areas (3)], vol. 26, Springer-Verlag, Berlin, 1993. MR1238941

[Väi66] Jussi Väisälä, Discrete open mappings on manifolds, Ann. Acad. Sci. Fenn. Ser. A I No. 392 (1966), 10. MR0200928

Department of Mathematics and Statistics, P.O. Box 35, FI-40014 University of Jyväskylä, Finland; and Department of Mathematical Analysis, Charles University in Prague, Sokolovska 83, Praha 8, 186 75, Czech Republic

Email address: rami.luisto@gmail.com 